\title{
An Estimate of the Prevalence of Dementia in Idiopathic Parkinson's Disease
}

Richard Mayeux, MD; Yaakov Stern, PhD; Ruth Rosenstein, MPH; Karen Marder, MD; Allan Hauser, MD; Lucien Cote, MD; Stanley Fahn, MD

- A review of the records for evidence of dementia using criteria adapted from the third edition of the Diagnostic and Statistical Manual of Mental Disorders in every patient (hospitalized and outpatient) with parkinsonism at a major medical center during an 18-month period revealed an overall prevalence of $10.9 \%$ in 339 patients with idiopathic Parkinson's disease. Demented patients were older, had a later age at onset of motor manifestations, and a more rapid progression of physical disability than nondemented patients. Duration of illness and levodopa use and the presence of tremor or depression were similar in demented and nondemented patients. Demented patients more often responded poorly or developed adverse effects to levodopa than nondemented patients. When Parkinson's disease began after age 70 years, dementia was noted over three times more frequently than when the disease began at an earlier age. The age-specific prevalence rate of dementia for patients older than $\mathbf{7 0}$ years was more than twice that for younger patients. Moreover, the number of records with evidence for dementia with idiopathic Parkinson's disease was 3.75 times greater than expected in comparison with data from a study of the prevalence of dementia in the elderly.

(Arch Neurol 1988;45:260-262)

The prevalence of dementia in Par1 kinson's disease (PD) is unsettled; estimates range from $14 \%$ to $40 \% .^{1}$ Brown and Marsden ${ }^{2}$ reviewed 17 studies reported over the last 60 years that included a total of 2530 patients with PD. Thirty-five percent of these patients had some degree of dementia, but the prevalence of dementia could have been overestimated because of sampling variability and differences

\footnotetext{
Accepted for publication Oct 13, 1987
}

From the Departments of Neurology (Drs Mayeux, Stern, Marder, Hauser, Cote, and Fahn and Ms Rosenstein), Psychiatry (Drs Mayeux and Stern), and Rehabilitation Medicine (Dr Cote), College of Physicians and Surgeons, Columbia University, New York.

Reprint requests to Neurological Institute, 710 W 168th St, New York, NY 10032 (Dr Mayeux). in criteria used for the diagnosis of dementia or idiopathic PD.

To estimate the frequency of dementia in $\mathrm{PD}$, we reviewed the records of over 422 patients with parkinsonism from an urban hospital population, using standardized research criteria for dementia and idiopathic $\mathrm{PD}$. Of the records with sufficient evidence to warrant a diagnosis of dementia, $10.9 \%$ of the 339 patients with idiopathic $\mathrm{PD}$ were demented. Compared with a data set from a standard population of elderly adults older than 60 years, the odds of developing dementia appear to be almost four times greater for patients with idiopathic PD. ${ }^{3}$

\section{SUBJECTS AND METHODS}

We reviewed the records of every patient (hospitalized or outpatient) with parkinsonism in a major teaching hospital seen on all services during the 18 months between March 1, 1983, and Sept 30, 1984. The population included both private and clinic patients. Although the hospital is a tertiary care institution, $90 \%$ of the patients came from the greater New York area and were followed up annually. The population was representative of a large urban hospital-based cohort. When a patient had more than one visit (hospital and/or outpatient visits) during the study period, only the first encounter was used in estimating the prevalence of dementia. All available information, including that from subsequent chart entries, was used to substantiate a diagnosis regardless of when it was recorded, however.

\section{Data Extraction}

A systematic data collection form to extract clinical information from records was designed by us before the survey. Consensus on criteria for the diagnoses to be described below was reached before the study, and the data form was used in a brief pilot survey before the formal data extraction began.

\section{Diagnoses}

Criteria for the diagnosis of idiopathic PD included the presence of at least two of the four characteristic motor manifestations of the disorder (tremor, shuffling gait, bradykinesia, or muscular rigidity) with a history of insidious onset. Patients with parkinsonism, resulting from encephalitis, medications, or multiple stroke, were eliminated from further consideration, as were patients with progressive supranuclear palsy.

We based our research diagnostic criteria for primary degenerative dementia and major depression on the definitions in the third edition of the Diagnostic and Statistical Manual of Mental Disorders (DSMIII). ${ }^{4}$ Briefly, we defined dementia as a sustained impairment in intellectual function, including memory, that was not considered drug related and occurred without an alteration in consciousness. This was done by reviewing subsequent chart entries to note whether the mental impairment, if present, was sustained or transient. We required a written description of a mental status examination by a neurologist or psychiatrist in the record that included an assessment of memory, orientation, and general knowledge. For dementia, all mental functions listed above had to be defective; disorientation alone was considered insufficient criteria for this diagnosis. In some patients quantitative neuropsychological data were present, allowing a more precise diagnosis. The diagnosis of dementia or idiopathic PD was retracted only if it was later refuted, in the record, by a qualified physician, such as a neurologist or psychiatrist. This is similar to the method used by Rajput et al. ${ }^{5}$ We defined depression as a dysphoric mood characterized by feelings of sadness and associated with changes in appetite and sleep habits.

We also recorded other features of PD: age at onset (defined as the time that the diagnosis of PD was first made by a qualified physician) and duration of $\mathrm{PD}$ motor manifestations (defined as the period from the onset of the illness to the first encounter recorded during the study period). In almost half of the patients, disease severity, including the presence or absence of dementia or depression, had been rated on the Columbia University PD rating scale. ${ }^{\circ}$ Ability to perform activities of daily living (ADL) was also usually rated. ${ }^{7}$ The disease was classified as tremulous or nontremulous type, depending on the presence or absence of a resting tremor. Response to levodopa therapy was ascertained: patients were classified as responders (good response to average dosages), secondary nonresponders (ie, good response at first but later not adequate despite sufficient 
dosage), or primary nonresponders (never had a good response). Duration of symptoms before and after levodopa therapy was started, as well as current total daily dose, was noted.

We estimated the rate of progression of $\mathrm{PD}$ by dividing patients into three groups at the time of chart review: benign (ADL score $>70 \%$ after five years of illness), average (ADL, $50 \%$ to $70 \%$ after three years), and rapid (ADL $<50 \%$ within one year of illness). Since some patients were hospitalized, we systematically recorded the reason for hospitalization because of its potential influence on our data.

Almost half of the data extraction forms were completed by clinicans at or near the time of an office visit. The other half, which included all of the hospitalized patients, were completed after chart review by two other clinicians (R.R. and R.M.). We compared the results of the data retrospectively with those collected at the office visit. In addition, three of us independently rereviewed 59 selected records. This second review consisted of randomly selected records from the demented patients matched to nondemented patients. Interrater agreement about the presence or absence of depression and dementia and a comparison with the previously recorded diagnoses were assessed by a $x$-statistic ${ }^{8}$ that is chance corrected.

\section{Comparison Group}

Because we wanted to estimate the frequency of dementia expected at this age, we used prevalence data from the Baltimore Longitudinal Study (BLS) ${ }^{3}$ for comparison. That study included volunteers older than the age of 60 years. By multiplying the number of patients with PD in each age group by the age-specific prevalence rate observed in the BLS we could estimate the number of cases of dementia that might have been expected in people older than the age of 70 years.

\section{RESULTS}

The records of 422 patients with parkinsonism were reviewed; of these, 355 were judged to have idiopathic PD. Sixteen of the patients with idiopathic $P D$ had the onset of motor symptoms before the age of 40 years and were excluded from further analysis because of the unusual age of onset. This left 339 patients in the study: $264(78 \%)$ were outpatients and $75(22 \%)$ were hospitalized during the study period. A mental status examination was not recorded in three patients, but their data were still included in the study without a diagnosis regarding dementia to maintain a conservative prevalence estimate.

Of the 75 hospitalized patients, 50 were admitted for various problems related to $P D$, such as medication adjustments or drug trials. Another 25 were hospitalized for medical or surgical problems unrelated to PD,

\begin{tabular}{|cccccc|}
\hline \multicolumn{5}{|c|}{$\begin{array}{c}\text { Prevalence of Dementia in Parkinson's Disease (PD) Compared With } \\
\text { Baltimore Longitudinal Study Sample }\end{array}$} \\
\hline Group & $n$ & Observed & Expected & SMR $^{*}$ & $95 \%$ Cl (SMR) \\
\hline $\begin{array}{c}\text { All patients with PD } \\
\text { older than 60 y }\end{array}$ & 272 & 36 & 9.38 & 3.84 & $(2.69,5.19)$ \\
\hline PD onset before 70 y & 221 & 23 & 4.79 & 4.80 & $(3.04,6.95)$ \\
\hline PD onset after 70 y & 51 & 13 & 4.75 & 2.73 & $(1.45,4.43)$ \\
\hline
\end{tabular}

- SMR indicates standardized morbidity ratio.

† $95 \% \mathrm{Cl}$ (SMR) indicates 95\% confidence interval for the SMR.

such as myocardial infarction or pneumonia.

We found sufficient evidence for dementia in the records of $37(10.9 \%)$ of the 339 study patients with PD seen during the prevalence period. Dementia was recorded in the charts of 18 (24\%) of 75 hospitalized patients and $19(7 \%)$ of 264 outpatients ( $z=3.95$, $P<.001)$. Demented patients were significantly older $(74.03 \pm 8.17$ years vs $66.91 \pm 8.92$ years; $P<.01)$, had a later age at onset of the motor manifestations of PD $(65.28 \pm 10.43$ years vs $59.25 \pm 9.67$ years; $P<.01)$, had more severe manifestations (measured by the Columbia University PD rating scale ${ }^{6}: 50.32 \pm 19.27$ vs $31.46 \pm$ $18.12, P<.01$ ), and were significantly more disabled than the patients without dementia (measured by the Schwab and England ${ }^{7}$ ADL scale: $44.0 \% \pm 21.97 \%$ vs $74.55 \% \pm 20.39 \%$, $P<.01)$. The proportion of demented patients whose motor manifestations began after the age of 70 years was significantly greater than the proportion of demented patients whose motor manifestations began at an earlier age ( $<69$ years, 23 [8.5\%] of 272 ; $>70$ years, 14 [20.9\%] of 67 : $z=2.933, \quad P<.001$ ). Demented patients more often had a rapid progression of disability than nondemented patients $\left(\chi^{2} 4.48, P<.05\right)$. Duration of illness, levodopa use and mean dosage, type of PD (tremulous vs nontremulous), and prevalence of depression (47\% in the total sample) were similar in demented and nondemented patients. However, the frequency of demented patients classified as either primary or secondary nonresponders to dopaminergic medications and the number developing adverse effects at relatively low doses was significantly greater than in the nondemented group $\left(\chi^{2} 9.41, P<.01\right)$. Onset of PD (motor manifestations) after age 70 years occurred more often in the demented than in the nondemented patients $\left(\chi^{2} 11.41, P<.01\right)$; the prevalence odds ratio for dementia in patients with onset of $P D$ after the age of 70 years compared with those with onset before the age of 70 years was 3.75 (95\% confidence interval, $1.75,7.95)$.

Thirty-seven patients had taken the Modified Mini-Mental State Examination at the time of their entry into the prevalence survey. Although the number of patients tested was small, the results agreed with the chart reviews. There were 33 nondemented patients (mean score, 51.0) and four demented patients (mean score, 24.5) $(t=4.33, P<.02)$. The total score for the Modified Mini-Mental State Examination is 57, and mean for elderly control subjects is $52.3 \pm 17$.

No differences were apparent in the prevalence of dementia, age of onset, or duration of illness when the information was collected near the time of an office visit or extracted at a later date from the record. Interrater agreement for the diagnosis of dementia from the medical records was $91 \%$ between the three independent reviewers. After correcting for chance agreement, the $\kappa$-statistic ${ }^{8}$ indicated that the agreement between the reviewers was $88 \%$. The agreement between these reviewers and the originally collected data was also high: $87 \%$.

To compare the prevalence of dementia in our study with a standard population we used the data from the BLS. ${ }^{3}$ We compared the prevalence of dementia in the 272 patients older than 60 years with idiopathic PD from our study with that data set; dementia was found 3.84 times more often than expected for that age group (Table). When PD began before the age of 70 years, dementia was noted in the records 4.8 times more often than would have been expected based on the BLS data, but when PD began after the age of 70 years, dementia was recorded 2.73 times more often than expected.

\section{COMMENT}

Using standardized criteria for dementia and for idiopathic PD, we found evidence of dementia in the records of $10.9 \%$ of patients with $\mathrm{PD}$. While this may support the claim that the overall prevalence of dementia in 
PD has been overestimated, ${ }^{2,9,10}$ it must be realized that we also found age-specific prevalence rates to increase after age 60 years. The retrospective and cross-sectional nature of the chart review makes it likely that we identified only severe dementia and underestimated the prevalence of mild dementia. We also may have slightly underestimated dementia by determining rates based on the first encounter during the prevalence period. However, our results do indicate that dementia in patients with $\mathrm{PD}$ is almost four times more frequent than expected in people older than the age of 60 years.

Although the prevalence of dementia in the general population increases with advanced age, the standardized morbidity ratios indicate that the prevalence of dementia in this cohort with idiopathic $P D$ is almost five times that expected for healthy people younger than the age of 70 years and almost three times greater than expected for healthy people older than 70 years. The reduction in the standardized morbidity ratio after the age of 70 years probably reflects the increased prevalence of dementia in the general population at that age. The comparison of our data to the $\mathrm{BLS}^{3}$ is only speculative because of major methodologic differences. Nevertheless, it implies that patients with idiopathic PD are at greater risk for dementia than is the general population regardless of when the motor manifestations begin.

In this study we found demented pa-

1. Mayeux R: Depression and dementia in Parkinson's disease, in Marsden CD, Fahn $\mathrm{S}$ (eds): Movement Disorders. Woburn, Mass, Butterworths, 1982, pp 75-95.

2. Brown RG, Marsden CD: How common is dementia in Parkinson's disease? Lancet 1984; 1:1262-1265.

3. Sayetta RB: Rates of senile dementia: Alzheimer's type in the Baltimore Longitudinal Study. J Chronic Dis 1986;39:271-286.

4. American Psychiatric Association, Committee on Nomenclature and Statistics: Diagnostic and Statistical Manual of Mental Disorders, ed 3. Washington, DC, American Psychiatric Association, 1980, pp 205-224.

5. Rajput AH, Offord KP, Beard CM, et al: A case-control study of smoking habits, dementia, and other illnesses in idiopathic Parkinson's disease. Neurology 1987;37:226-232.

6. Lesser RP, Fahn S, Snider SR, et al: Analysis of the clinical problems in parkinsonism and the complications of long-term levodopa therapy. Neurology 1979;29:1253-1260.

7. Schwab JF, England AC: Projection technique for evaluating surgery in Parkinson's disease, in Gillingham FJ, Donaldson MC (eds): Third Symposium on Parkinson's Disease. Edinburgh, E \& S Livingstone, 1969, pp 152-157.

8. Fleiss JL: Statistical Methods for Rates and Proportions. New York, John Wiley \& Sons Ine, 1981, pp 212-236. tients with PD to be older than nondemented patients and to have a more rapid progression of illness and greater disability, as others have found..$^{11-13}$ Although duration of $\mathrm{PD}$ is similar in demented and nondemented patients, the onset of dementia may reduce survival. Mindham et $\mathrm{al}^{14}$ reported that four of 16 demented patients died within three years, compared with two of the 24 nondemented patients.

Estimates of the prevalence of intellectual decline, including dementia, in PD vary considerably.' Brown and Marsden ${ }^{2}$ consider this variation to be due to differences in methods, such as the use of nonstandardized criteria for dementia and PD. Three separate studies of dementia in $\mathrm{PD}^{11-13}$ used similar mental status assessments. Adjusting the prevalence rates to satisfy $D S M-I I I$ criteria for dementia indicates that about $15 \%$ of the almost 1000 patients in these three studies were demented. This still suggests that the prevalence of dementia is greater than in control subjects, and Lees ${ }^{9}$ and Taylor et al ${ }^{10}$ support this amended prevalence data. In one Northampton region of the United Kingdom, the prevalence of idiopathic PD was 108.4 per 100000 , and $11 \%$ of the 223 patients with idiopathic PD were demented by a reasonable assessment of mental function and disability although there were no control subjects. ${ }^{15}$ However, in our study we found the age-specific rates of dementia in PD to increase significantly after the age of 70 years, as did Marttila and Rinne. ${ }^{13}$

\section{References}

9. Lees AJ: Parkinson's disease and dementia. Lancet 1985;1:43-44.

10. Taylor A, Saint-Cyr JA, Lang AE: Dementia prevalence in Parkinson's disease. Lancet 1985;1:1037.

11. Celesia GG, Wanamaker WM: Psychiatric disturbances in Parkinson's disease. Dis Nerv Syst 1972;33:577-583.

12. Lieberman A, Dziatolowski M, Coopersmith M, et al: Dementia in Parkinson's disease. Ann Neurol 1979;6:355-359.

13. Marttila RJ, Rinne UK: Dementia in Parkinson's disease. Acta Neurol Scand 1976;54:431441.

14. Mindham RHS, Ahmed SWA, Clough CG: A controlled study of dementia in Parkinson's disease. J Neurol Neurosurg Psychiatry 1982; 45:969-974.

15. Sutcliffe RLG: Parkinson's disease in the district of the Northampton Health Authority, United Kingdom: A study of prevalence and disability. Acta Neurol Scand 1985;72:363-379.

16. Boller F, Mizutani T, Roessmann U, et al: Parkinson's disease, dementia, and Alzheimer's disease: Clinicopathological correlations. $A n n$. Neurol 1980;1:329-335.

17. Mayeux R, Stern $Y$, Rosen $J$, et al: Is 'subcortical dementia' a recognizable clinical entity? Ann Neurol 1983;14:278-283.

18. Ruberg M, Ploska A, Javoy-Agid F, et al: Muscarinic binding and choline acetyltransfer-
Rajput et $\mathrm{al}^{\mathrm{s}}$ found that the probability of developing dementia was greater in patients with idiopathic $\mathrm{PD}$ than in a comparison group of similarly aged adults. The cumulative probability of dementia was $21.1 \%$ for patients with PD compared with 5.7\% for control subjects after five years.

Because there is a remarkable overlap in the clinical, ${ }^{16,17}$ biochemical, ${ }^{18}$ and pathological $1^{19-21}$ manifestations of dementia in PD and in Alzheimer's disease, the cause of dementia in PD is of interest. Idiopathic PD may predispose to dementia. However, the only known risk factor is a late age at disease onset. In addition, some types of intellectual impairment in PD do not warrant a diagnosis of dementia, $22-25$ and postmortem studies are often inconclusive.

Using $D S M-I I I^{4}$ criteria, it is apparent that most patients with idiopathic PD are not demented. Our data must be interpreted with caution because we may have identified only severe dementia, they were collected retrospectively, and we used an external comparison group. Nevertheless, our results suggest that while the prevalence of dementia is relatively low, the odds of becoming demented with idiopathic PD, compared with a standard population, may be significantly increased.

This investigation was supported by Public Health Service grant AG 02802 (Dr Mayeux) and a grant from the Parkinson's Disease Foundation, New York. Data were stored and analyzed on a CLINFO system (RR00654) of the Public Health Service.

ase activity in parkinsonian subjects with reference to dementia. Brain Res 1982;232:129-133.

19. Alvord EC, Forno LS, Kusske JA, et al: The pathology of parkinsonism: A comparison of degeneration in cerebral cortex and brain stem. Adv Neurol 1975;5:175-193.

20. Forno LS, Alvord EC: The pathology of parkinsonism: I. Some new observations, in McDowell F, Markham C (eds): Recent Advances in Parkinson's Disease. Philadelphia, FA Davis Co Publishers, 1971, vol 8, pp 120-130.

21. Forno LS: Pathology of Parkinson's disease, in Marsden CD, Fahn S (eds): Movement Disorders. Woburn, Mass, Butterworths, 1982, pp 25-40.

22. Matison R, Mayeux R, Rosen $J$, et al: 'Tip-of-the-tongue' phenomenon in Parkinson's disease. Neurology 1982;32:567-570.

23. Matthews CG, Haaland KY: The effect of symptom duration on cognitive and motor performance in parkinsonism. Neurology 1979; 29:951-956.

24. Stern Y, Mayeux R, Rosen J, et al: Perceptual motor dysfunction in Parkinson's disease: A deficit in sequential and predictive movement. $J$ Neurol Neurosurg Psychiatry 1983;46:145-151.

25. Wilson RS, Kaszniak AW, Klawans HL, et al: High-speed memory scanning in parkinsonism. Cortex 1980;16:67-72. 